\title{
EVALUACIÓN DE MEZCLAS DE ARCILLAS PARA LA FABRICACIÓN DE LADRILLOS REFRACTARIOS QUE SIRVAN PARA LA RECONVERSIÓN TECNOLÓGICA DE LOS HORNOS UTILIZADOS EN NORTE DE SANTANDER
}

\author{
EVALUATION OF MIXTURES OF CLAY FOR THE MANUFACTURE OF REFRACTORY \\ BRICKS USED FOR THE CONVERSION TECHNOLOGY OF USED OVENS \\ IN NORTH SANTANDER
}

Jorge Sánchez Molina ${ }^{1}$, Julia Andrea Orozco Cacique² ${ }^{2}$ Leidy Peñaloza Isidro ${ }^{3}$

1. Ingeniero Químico UIS. Magister en Gerencia de empresas mención industria UNET. Centro de Inves tigación de Materiales Cerámicos, Grupo de investigación en tecnología cerámica GITEC, Universidad Francisco de Paula Santander, Cúcuta-Colombia. E-mail: j_sanchezmolina@yahoo.es.

2. Ingeniera Industrial UFPS. Estudiante de maestría en calidad y gestión integral USTA. Grupo de investigación en tecnología cerámica GITEC, Universidad Francisco de Paula Santander, CúcutaColombia. E-mail: andri orozco@hotmail.com.

3. Ingeniera Industrial UFPS. Centro de Investigación de Materiales Cerámicos, Universidad Francisco de Paula Santander, Cúcuta-Colombia. E-mail: ydiel_0304@hotmail.com

Recibido: 30 de Julio de 2014

Aceptado: 01 de Noviembre de 2014

*Correspondencia del Autor: Centro de Investigación de Materiales Cerámicos, Grupo de investigación en tecnología cerámica GITEC, Universidad Francisco de Paula Santander, Cúcuta-Colombia. E-mail: j_sanchezmolina@yahoo.es.

\section{RESUMEN:}

Se analizaron las propiedades físicas (aptitud cerámica) y químicas (Fluorescencia de rayos X FRX) de cuatro materias primas (arcilla Peracos, arcilla La Alejandra, Caolín HS-801 A y mezcla Táchira), logrando obtener indicadores de medición a través de las temperaturas estudiadas $\left(1150\right.$ y $\left.1280^{\circ} \mathrm{C}\right)$, para identificar su composición y el comportamiento individual; y de esta manera seleccionar las cantidades a ser empleadas en la formulación de las diferentes mezclas: MA, MB, MC, MD, ME y MF. Finalmente se emplearon las mezclas más apropiadas para la fabricación de un prototipo de ladrillo refractario a escala real, siendo la mezclas MA con un bajo porcentaje de contracción en cocido de 2,89 a la temperatura de $1280 \mathrm{oC}$, una densidad aparente constante de $1,60 \mathrm{~g} / \mathrm{cm}^{3} \mathrm{y}$ una resistencia a la rotura de $16,98 \mathrm{Kgf} / \mathrm{cm}^{2}$, MB con un bajo porcentaje de contracción en cocido de 5,69 a la temperatura de $1280^{\circ} \mathrm{C}$, una densidad aparente $1,58 \mathrm{~g} / \mathrm{cm}^{3}$ y una resistencia a la rotura de $49,01 \mathrm{Kgf} / \mathrm{cm}^{2}$, y ME con un bajo porcentaje de contracción en cocido de 5,88 a la temperatura de $1280^{\circ} \mathrm{C}$, una densidad aparente 0,93 $\mathrm{g} / \mathrm{cm}^{3}$ y una resistencia a la rotura de $7,68 \mathrm{Kgf} / \mathrm{cm}^{2}$; las que cumplen con las condiciones requeridas por la NTC 623 para la fabricación de ladrillos refractarios.

Palabras Claves: Arcilla, Caolín, ladrillo refractario, caracterización de materias primas. 


\begin{abstract}
:
The physical (ceramic aptitude) and chemical (X-ray fluorescence) properties of four raw materials were analyzed (Peracos clay, La Alejandra clay, Kaolin HS-801 A and mixes Táchira). Measurement indicators were obtained through the studied temperatures $\left(1150\right.$ and $\left.1280^{\circ} \mathrm{C}\right)$ to identify its composition and the individual behavior; and select the amounts to be used in the formulation of the different mixtures: MA, MB, MC, MD, ME and MF. Finally, the most appropriate mixtures for the manufacture of a refractory brick prototype were used to real scale. The mixture MA presented a low percentage of contraction in stew of 2.89 at the temperature of $1280{ }^{\circ} \mathrm{C}$, a constant apparent density of $1,60 \mathrm{~g} / \mathrm{cm} 3$ and resistance to the break of $16.98 \mathrm{Kgf} / \mathrm{cm} 2$. MB presented a low percentage of contraction in stew of 5.69 to the temperature of $1280{ }^{\circ} \mathrm{C}$, an apparent density of $1.58 \mathrm{~g} / \mathrm{cm} 3$ and a resistance to the break of $49.01 \mathrm{Kgf} / \mathrm{cm} 2$, and $\mathrm{ME}$ presented a low percentage of contraction in stew of 5,88 to the temperature of $1280{ }^{\circ} \mathrm{C}$, an apparent density of $0.93 \mathrm{~g} / \mathrm{cm} 3$ and a resistance to the break of $7,68 \mathrm{Kgf} / \mathrm{cm} 2$; those that fulfill the conditions required by NTC 623 for the refractory brick manufacture.
\end{abstract}

Keywords: Clay, Kaolin, refractory brick, characterization of raw materials.

\section{INTRODUCCIÓN.}

En el Departamento de Norte de Santander, Colombia se encuentran gran variedad de materias primas arcillosas, lo que ha permitido la consolidación del sector cerámico, el cual está conformado en su mayoría por empresas dedicadas a la producción de materiales de construcción, siendo este Departamento el principal productor de baldosas cerámicas no esmaltadas a nivel nacional, dada las excelentes características de las materias primas $(1,2)$.

Los refractarios son materiales utilizados para procesos de alta temperatura, cuyas características principales son: elevado punto de fusión, capacidad para almacenar calor, capacidad aislante y buena resistencia físico-química.

El sector ladrillero de Cúcuta y su área metropolitana emplea hornos colmena en un $81,8 \%$, los cuales generan graves problemas ambientales por emisiones atmosféricas debido a la baja eficiencia energética y a las grandes cantidades de combustible orgánico (carbón) requeridas, así como la baja vida útil de los hornos por lo que deben repararse frecuentemente. Para la construcción de hornos la elección del refractario debe ser acertada ya que éste debe adaptarse al trabajo que se realiza, se debe tener en cuenta la expansión térmica (dilatación) y que sea tan buen aislante como sea posible. Así mismo, debido a las exigencias del ciclo térmico asociado a la cocción de artículos cerámicos se deben tener en cuenta características como la absorción de agua, resistencia a la flexión, contracción en cocido, densidad aparente y porosidad aparente.

Teniendo en cuenta, que algunas empresas de la región, se han concientizado de la necesidad de un cambio de tecnología en su proceso, principalmente en los hornos se considera importante contar con ladrillos refractarios fabricados en la región que se ajusten a sus necesidades de las empresas del sector, con este fin se desarrolló esta investigación.

MATERIALES Y MÉTODOS. Para el desarrollo de la investigación se inició con la selección de materias primas de la región y sus alrededores muestreadas en planta, se llevó a cabo la caracterización de las materias primas a través de la aplicación de los siguientes ensayos: contracción en cocido, pérdidas por calcinación, absorción de agua y densidad aparente, los cuales fueron realizados según métodos internacionales y del libro: Manual para el control de la calidad de materias primas arcillosas (3).

Luego se realizaron, seis formulaciones con las materias primas caracterizadas, y posteriormente con estas mezclas se elaboraron probetas prensadas de forma rectangular (110 $\mathrm{mm}$ de largo, $55 \mathrm{~mm}$ de ancho, 0,8 $\mathrm{mm}$ de espesor) a nivel de laboratorio, mediante la aplicación las etapas del proceso productivo para fa- 
bricación de ladrillo refractario, dichas probetas fueron caracterizadas, y finalmente se seleccionaron las mezclas más apropiadas para la fabricación de un pro-

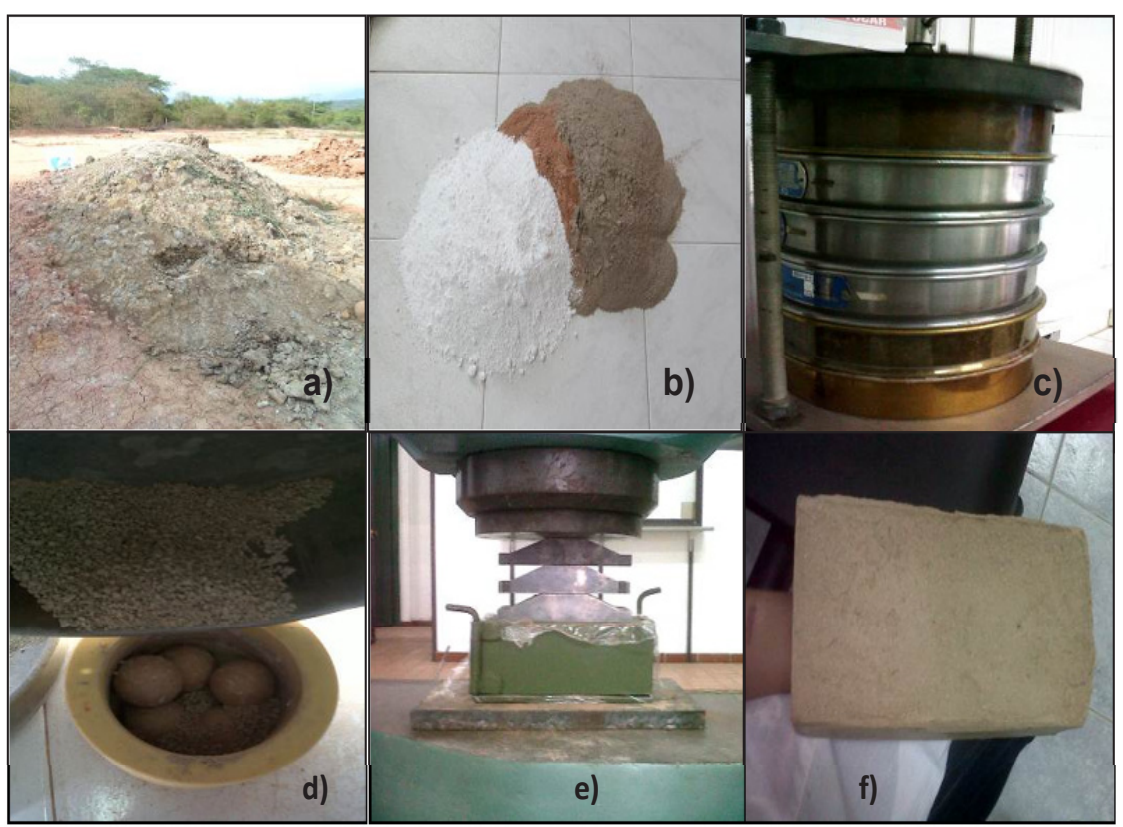

Figura 1. Proceso productivo del ladrillo refractario. a) Extracción de materias primas, b) preparación de mezclas, c) Tamizado, d) Molienda, e) Prensado en seco, f) Ladrillo refractario a escala real

Materias primas. Se realizó una selección previa de diferentes materias primas de la región, y algunas de otras regiones que cuentan con las características químicas necesarias para la fabricación de un ladrillo refractario $(4,5,6)$. Posteriormente se caracterizaron a través de ensayos de laboratorio

Las materias primas de la región analizadas fueron: arcilla PERACOS ubicada en el municipio de San Faustino, arcilla LA ALEJANDRA ubicada en el corregimiento La Alejandra, El Zulia; y fuera de la región se caracterizó CAOLÍN HS-801A ubicado en Medellín, Antioquia y finalmente la arcilla MEZCLA TÁCHIRA ubicada en el Estado Táchira en La República Bolivariana de Venezuela.

Formulación de mezclas. Para la formulación de las mezclas se tuvo en cuenta la norma NTC 623, la cual expresa que los refractarios adecuados para las temperaturas de cocción y tipo de productos a obtener, deben poseer una relación de alúmina $\left(\mathrm{Al}_{2} \mathrm{O}_{3}\right)$ y sílice
$\left(\mathrm{SiO}_{2}\right)$ lo suficientemente alta para elevar su punto de fusión. Las formulaciones se han desarrollado teniendo en cuenta que los ladrillos poco refractarios tienen un punto de cono equivalente $\mathrm{PCE}$, Cono $\mathrm{N}^{\circ} 15$ (1430 ${ }^{\circ} \mathrm{C}$ ) y deben presentar una composición química de alúmina entre 25 - $30 \%$, y mayor de 90\% entre Alúmina y Sílice $(7,8,9,10)$.

Una vez atendidas estas condiciones se procedió a preparar seis mezclas. Para la formulación de las diferentes mezclas se tomó como partida los análisis químicos de las materias primas, de tal manera que los porcentajes de alúmina $\left(\mathrm{Al}_{2} \mathrm{O}_{3}\right)$ sean mayores a 25 $\%$. Posteriormente se elaboraron mezclas con las formulaciones definidas de tal manera que la cantidad presente de arcilla variaba en las diferentes formulaciones, con el fin de obtener varias opciones para que se cumpliera con los requisitos deseados. En cada mezcla se empleó al menos una de las dos arcillas seleccionadas en la región. 
Tabla 1. Formulación de mezclas

\begin{tabular}{lcccccccc}
\hline MEZCLA & $\begin{array}{c}\text { PERACOS } \\
(\%)\end{array}$ & $\begin{array}{c}\text { LA ALEJANDRA } \\
(\%)\end{array}$ & $\begin{array}{c}\text { CAOLIN HS 801 } \\
(\%)\end{array}$ & $\begin{array}{c}\text { CHAMOTA } \\
(\%)\end{array}$ & $\begin{array}{c}\text { MEZCLA TA- } \\
\text { CHIRA (\%) }\end{array}$ & $\begin{array}{c}\mathbf{A l}_{2} \mathbf{O}_{3} \\
(\%)\end{array}$ & $\begin{array}{c}\mathbf{S i O}_{2} \\
(\%)\end{array}$ & $\begin{array}{c}\mathrm{Al}_{2} \mathbf{O}_{3}+\mathrm{SiO}_{2} \\
(\%)\end{array}$ \\
\hline MA & 30,00 & 10,00 & 0,00 & -- & 60,00 & 35,34 & 58,22 & 93,56 \\
MB & 0,00 & 70,00 & 0,00 & -- & 30,00 & 25,73 & 61,82 & 87,54 \\
MC & 30,00 & 20,00 & 5,00 & -- & 45,00 & 32,84 & 59,54 & 92,37 \\
MD & 70,00 & 10,00 & 20,00 & -- & -- & 27,86 & 64,01 & 91,86 \\
ME & 30,00 & 20,00 & 50,00 & -- & -- & 33,52 & 59,44 & 92,96 \\
MF & 30,00 & 20,00 & 40,00 & 10,00 & -- & 30,80 & 60,79 & 91,59 \\
\hline
\end{tabular}

Elaboración de prototipos a nivel laboratorio. Existen dos métodos en la fabricación de ladrillo refractario, conformado por extrusión y conformado por prensado, sin embargo para el desarrollo de la investigación se realizaron los ladrillos refractarios por prensado debido a que el producto terminado presenta un mejor aspecto.

Para fabricar ladrillos por prensado se aconseja un control en la determinación de la granulometría de la mezcla, mediante un tamizado. (5)

Además es importante tener un control en la presión, es decir, si está es muy baja el producto puede tener baja densidad o baja resistencia mecánica, y si es muy alta el material puede sufrir laminaciones o grietas de prensado.
Una vez seleccionado el método de conformado, se trituraron las materias primas, se molieron y se tamizaron por malla ASTM 18 para luego ser mezcladas dándoles el grado de humectación requerida así como el tamaño de partícula óptimo, posteriormente se realizó el conformado por prensado en una prensa hidráulica a una presión de $240 \mathrm{Kgf} / \mathrm{cm}^{2}$ y en una proporción de 100 gramos por probeta. Se tomaron condiciones como peso y dimensiones de las probetas en húmedo.

Caracterización de probetas. Después de prensadas las probetas se colocaron en un secadero a una temperatura de $110{ }^{\circ} \mathrm{C}$ durante 24 horas, después se tomaron sus condiciones en seco, seguidamente son llevadas al horno mufla donde se realiza la cocción de las probetas en forma gradual hasta alcanzar la temperatura de-

Tabla 2. Características de las materias primas empleadas

\begin{tabular}{|c|c|c|c|c|c|}
\hline \multicolumn{2}{|c|}{$\begin{array}{c}\text { CARACTERISTICA } \\
\% \text { peso }\end{array}$} & PERACOS & LA ALEJANDRA & CAOLÍN HS-801 A & MEZCLA TACHIRA \\
\hline \multirow{10}{*}{$\begin{array}{l}\text { Composición } \\
\text { química }\end{array}$} & $\left(\% \mathrm{~A}_{1} 2 \mathrm{O}_{3}\right)$ & 24,29 & 18,0 & 45,27 & 43,75 \\
\hline & $\left(\% \mathrm{SiO}_{2}\right)$ & 67,09 & 65,8 & 52,31 & 52,52 \\
\hline & $\left(\% \mathrm{Fe}_{2} \mathrm{O}_{3}\right)$ & 5,21 & 6,0 & 0,50 & 0,85 \\
\hline & $\left(\% \mathrm{TiO}_{2}\right)$ & 0,91 & 0,96 & 0,80 & 2,12 \\
\hline & $\left(\% \mathrm{Na}_{2} \mathrm{O}\right)$ & 0,45 & 0,1 & 0,20 & 0,52 \\
\hline & $\left(\% \mathrm{~K}_{2} \mathrm{O}\right)$ & 1,14 & 1,7 & 0,10 & 0,23 \\
\hline & $(\% \mathrm{CaO})$ & 0,43 & 0,3 & 0,50 & 0 \\
\hline & $(\% \mathrm{MgO})$ & 0,52 & 0,7 & 0,30 & 0,01 \\
\hline & $\left(\% \mathrm{P}^{2} \mathrm{O}_{5}\right)$ & 0 & 0,11 & 0 & 0 \\
\hline & $\left(\% \mathrm{SO}_{3}\right)$ & 0 & 0,11 & 0 & 0 \\
\hline \multirow{2}{*}{$\begin{array}{l}\text { Contracción } \\
\text { en cocido }\end{array}$} & $1150^{\circ} \mathrm{C}$ & 3,61 & 2,73 & 3,33 & 0,84 \\
\hline & $1280^{\circ} \mathrm{C}$ & 5,53 & 4,36 & 7,1 & 1,35 \\
\hline \multirow{2}{*}{$\begin{array}{l}\text { Pérdidas por } \\
\text { calcinación }\end{array}$} & $1150^{\circ} \mathrm{C}$ & 5,82 & 5,86 & 0,95 & 5,67 \\
\hline & $1280^{\circ} \mathrm{C}$ & 6,08 & 6,00 & 1,57 & 6,42 \\
\hline \multirow{2}{*}{$\begin{array}{l}\text { Absorción } \\
\text { de agua }\end{array}$} & $1150^{\circ} \mathrm{C}$ & 16,2 & 10,2 & 8,48 & 17,88 \\
\hline & $1280^{\circ} \mathrm{C}$ & 12,85 & 7,05 & 3,48 & 14,32 \\
\hline \multirow{2}{*}{$\begin{array}{l}\text { Densidad } \\
\text { aparente }\end{array}$} & $1150^{\circ} \mathrm{C}$ & 2,23 & 2,18 & 1,16 & 1,89 \\
\hline & $1280^{\circ} \mathrm{C}$ & 2,38 & 2,30 & 1,30 & 1,91 \\
\hline
\end{tabular}


seada (1150- 1250 y $\left.1280^{\circ} \mathrm{C}\right)$. Una vez finalizado este proceso se toman las condiciones cocidas, realizando ensayos de contracción en cocido (CC), absorción de agua (AA), densidad aparente (DA), porosidad aparente (PA), resistencia a la flexión (RF) $(11,12)$

\section{RESULTADOS Y DISCUSIÓN}

\section{Caracterización de las materias primas}

En la tabla 2 se observa que la mezcla Táchira es la que posee la menor contracción en cocido, con un valor del $1,35 \%$ a $1280^{\circ} \mathrm{C}$, lo siguen las arcillas de la mina Alejandra y Peracos con 5,53\% y $4,36 \%$ respectivamente, a la misma temperatura, esto se fundamenta en que los ladrillos refractarios deben soportar altas temperaturas, con una baja variación en su volumen. Para este tipo de materiales resultan más atractivas materias primas con una baja contracción. (4). El caolín, es el material que posee un valor bajo de pérdidas por calcinación, (1,57\%), comparada con las demás materias primas. La absorción de agua es menor a la temperatura $1280{ }^{\circ} \mathrm{C}$, debido a que mayor temperatura hay mayor sinterización, lo que disminuye la porosidad abierta de los productos cocidos. La densidad aparente incrementa a mayor temperatura, siendo el material menos denso es el caolín.

Caracterización de las mezclas. La tabla 3 muestra las propiedades como, contracción lineal de secado (C.S) y contracción en cocción (C.C), densidad aparente (D.A), absorción de agua (A.A), Resistencia a la flexión (R.F), porosidad aparente (P.A) y cono pirométrico equivalente (PCE). Estas propiedades son muy utilizadas en la evaluación y comparación de la calidad del producto, como parte de los criterios de selección y usos de los productos refractarios en variedad de aplicaciones industriales $(13,14,15)$.

Tabla 3. Comparación de resultados.

\begin{tabular}{|c|c|c|c|c|c|c|c|}
\hline \multicolumn{2}{|c|}{ ENSAYOS } & \multirow{2}{*}{$\begin{array}{c}\text { C.S } \\
\%\end{array}$} & \multirow{2}{*}{$\begin{array}{c}\text { C.C } \\
\%\end{array}$} & \multirow{2}{*}{$\underset{\%}{\text { A.A }}$} & \multirow{2}{*}{$\begin{array}{c}\text { D.A } \\
\%\end{array}$} & \multirow{2}{*}{$\begin{array}{c}\text { R.F } \\
\%\end{array}$} & \multirow{2}{*}{$\begin{array}{c}\text { P.A } \\
\%\end{array}$} \\
\hline & $\mathrm{T}\left({ }^{\circ} \mathrm{C}\right)$ & & & & & & \\
\hline \multirow{3}{*}{ MA } & $1150^{\circ} \mathrm{C}$ & \multirow{3}{*}{0,10} & 1,42 & 18,54 & 1,60 & 8,64 & 28,79 \\
\hline & $1250^{\circ} \mathrm{C}$ & & 2,55 & 18,88 & 1,60 & 15,05 & 26,61 \\
\hline & $1280^{\circ} \mathrm{C}$ & & 2,89 & 18,98 & 1,60 & 16,98 & 25,96 \\
\hline \multirow{3}{*}{ MB } & $1150^{\circ} \mathrm{C}$ & \multirow{3}{*}{0,34} & 3,47 & 13,27 & 1,59 & 36,24 & 21,10 \\
\hline & $1250^{\circ} \mathrm{C}$ & & 5,18 & 11,59 & 1,58 & 46,06 & 20,85 \\
\hline & $1280^{\circ} \mathrm{C}$ & & 5,69 & 11,09 & 1,58 & 49,01 & 20,77 \\
\hline \multirow{3}{*}{ MC } & $1150^{\circ} \mathrm{C}$ & \multirow{3}{*}{$-0,30$} & 1,25 & 17,12 & 1,60 & 15,40 & 27,55 \\
\hline & $1250^{\circ} \mathrm{C}$ & & 3,23 & 15,30 & 1,60 & 26,80 & 24,03 \\
\hline & $1280^{\circ} \mathrm{C}$ & & 3,83 & 14,76 & 1,60 & 30,23 & 22,97 \\
\hline \multirow{3}{*}{ MD } & $1150^{\circ} \mathrm{C}$ & \multirow{3}{*}{0,20} & 9,21 & 10,80 & 2,15 & 32,10 & 23,18 \\
\hline & $1250^{\circ} \mathrm{C}$ & & 7,73 & 12,85 & 2,01 & 22,30 & 25,85 \\
\hline & $1280^{\circ} \mathrm{C}$ & & 7,85 & 12,84 & 1,48 & 22,43 & 19,03 \\
\hline \multirow{3}{*}{ ME } & $1150^{\circ} \mathrm{C}$ & \multirow{3}{*}{$-0,12$} & 0,17 & ---- & ---- & ---- & ---- \\
\hline & $1250^{\circ} \mathrm{C}$ & & 2,14 & 39,62 & 1,28 & 1,61 & 50,73 \\
\hline & $1280^{\circ} \mathrm{C}$ & & 5,88 & 30,95 & 0,93 & 7,86 & 28,64 \\
\hline \multirow{3}{*}{ MF } & $1150^{\circ} \mathrm{C}$ & \multirow{3}{*}{0,15} & 5,70 & 26,84 & 1,40 & 11,95 & 37,45 \\
\hline & $1250^{\circ} \mathrm{C}$ & & 5,91 & 27,10 & 1,32 & 11,58 & 35,86 \\
\hline & $1280^{\circ} \mathrm{C}$ & & 5,69 & 27,96 & 1,38 & 11,16 & 38,68 \\
\hline
\end{tabular}


La menor contracción de secado la presentó el prototipo de la mezcla MA arrojando un valor de $0,1 \%$, y la más alta fue presentada por la mezcla MB siendo $0,34 \%$ su valor.

La contracción en cocido es directamente proporcional al aumento de la temperatura dando mejores resultados ME y MA las cuales tienen una contracción menor en comparación con las demás mezclas, siendo un indicativo importante para la elaboración de los ladrillos refractarios, ya que a mayor temperatura se comportara mejor dentro del horno.

La absorción de agua y la densidad aparente se realizaron según la norma ICONTEC NTC 674 (16), se evidencia que los prototipos de las mezclas MD y MB son los que presentan menor absorción de agua, ya que fueron los que arrojaron mayor contracción de cocido; La densidad aparente en las mezclas MA y MC es constante, presentando un valor de $1,6 \mathrm{~g} / \mathrm{cm}^{3}$ a las tres temperaturas. Asimismo, se observa que a la temperatura de $1150^{\circ} \mathrm{C}$ la mezcla $\mathrm{MC}$ es la que tiene mayor densidad aparente comparada con las demás mezclas, y a $1280^{\circ} \mathrm{C}$ la mezcla $\mathrm{ME}$ es la de menor densidad aparente.

La resistencia a la rotura por flexión fue realizada mediante la norma ICONTEC NTC 4321-4 (17), se puede observar que a $1280^{\circ} \mathrm{C}$ la mezcla MB presenta un mejor comportamiento, ya que se obtuvieron valores altos. Por otra parte se evidencia que la ME presenta valores bajos de resistencia a la flexión, lo cual es un comportamiento esperado teniendo en cuenta la sinterización del material.

La mezcla MF presenta un comportamiento similar en las tres temperaturas, siendo su porosidad aparente mayor que las otras mezclas, por otra parte la $\mathrm{ME}$ presenta un valor de porosidad aparente muy elevado a la temperatura de $1250^{\circ} \mathrm{C}$ comparado con el valor arrojado a $1280^{\circ} \mathrm{C}$, debido a la baja sinterización del material

Finalmente se realizó el ensayo de cono pirométrico equivalente con la mezcla $\mathrm{A}$, con la mezcla $\mathrm{B}$ y con la mezcla C (Tabla 4).

Tabla 4. Cono Pirométrico Equivalente.

\begin{tabular}{ccc}
\hline MEZCLA & NUMERO DE CONO & TEMPERATURA OC \\
\hline MA & 15 & 1430 \\
MB & $13-14$ & $1349-1398$ \\
ME & 14 & 1398 \\
\hline
\end{tabular}

La determinación del (PCE) se realizó según norma ICONTEC NTC 706 (18). El cono equivalente precisa las características del material elaborado y la carga de temperatura que soporta. En este caso la mezcla MA (60\% Mezcla Táchira, Pera$\cos 30 \%$ y Alejandra 10\%) soporta una temperatura de $1430{ }^{\circ} \mathrm{C}$, la mezcla MB (70\% Alejandra y 30\% Mezcla Táchira) soporta una temperatura entre $1349{ }^{\circ} \mathrm{C}$ y $1398{ }^{\circ} \mathrm{C}$ y la mezcla $\mathrm{ME}(30 \%$ Peracos, 20\% Alejandra y 50\% de Caolín) soporta una temperatura de $1398{ }^{\circ} \mathrm{C}$.

\section{CONCLUSIONES.}

Los ladrillos refractarios fabricados durante esta investigación se consideran aptos para ser empleados en la construcción o reconstrucción de hornos tipo colmena, esto teniendo en cuenta que dichos ladrillos soportan una temperatura de $1430^{\circ} \mathrm{C}$ el de $\mathrm{MA}$ y $1349^{\circ} \mathrm{C}$ a $1398^{\circ} \mathrm{C}$ el de $\mathrm{MB}$ y los hornos colmena utilizados en Norte de Santander trabajan a temperaturas máximas de entre $950^{\circ} \mathrm{C}$ y $1080^{\circ} \mathrm{C}$.

De acuerdo a los datos obtenidos en los ensayos de contracción en cocido, absorción de agua, densidad aparente y resistencia a la rotura por flexión, se puede deducir que las mezclas MA (30\% arcilla Peracos- 10\% arcilla La Alejandra- 60\% Mezcla Táchira), MB (70\% arcilla La Alejandra- 30\% Mezcla Táchira) y ME (30\% de arcilla Peracos- 20\% arcilla La Alejandra- 50\% Caolín); cumplen con los parámetros y características para la fabricación de un ladrillo refractario.

El 81,8 \% de los hornos utilizados en la región son colmena, los ladrillos refractarios fabricados con las mezclas MA y MB se pueden usar en la cúpula, los sacos y las hornillas; aumentando la productividad ya que se disminuirían los tiempos de parada para el mantenimiento de los hornos. En los hornos túnel se pueden emplear estos ladrillos refractarios en la sección de precalentamiento y en la de enfriamiento. 


\section{AGRADECIMIENTO.}

Los autores expresan sus agradecimientos a COLCIENCIAS por el apoyo brindado a través del programa Jóvenes Investigadores e Innovadores, a la Ladrillera Sigma por su colaboración e interés y de manera especial a la UNIVERSIDAD FRANCISCO DE PAULA SANTANDER y al Centro de Investigación de Materiales Cerámicos, por su aporte en la ejecución de esta investigación.

\section{BIBLIOGRAFÍA}

1. Peñaloza Isidro L y Tolosa Franco J. Evaluación de mezclas de arcillas para la fabricación de ladrillos refractarios en el área metropolitana de Cúcuta. Universidad Francisco de Paula Santander. Facultad de Ingeniería. Trabajo de grado de Ingeniería Industrial. 2013.

2. Rozo Rincón S y Ramírez Gómez P. Estudio de factibilidad para la producción de ladrillos semirefractarios para la construcción y preparación de hornos de la industria cerámica en el Área Metropolitana de Cúcuta. Universidad Francisco de Paula Santander. Facultad de Ingeniería. Trabajo de grado de Ingeniería de Producción Industrial. 2009.

3. Amorós J, Sánchez E, García-Ten J et al. Manual para el control de la calidad de materias primas arcillosas. Instituto de Tecnología cerámica ITC. Castellón, España.1998.

4. Zúñiga Lázaro J; Ortega Julio. Estudio de la arcilla del Municipio de Abrego (Norte de Santander) y su utilización en la fabricación de refractarios. Cúcuta. Universidad Francisco de Paula Santander. Facultad de Tecnologías. Escuela de Tecnología Química. 1987.

5. Rivera R; Rodríguez A. Estudio de la ceniza del carbón en la elaboración de ladrillos refractarios. Cúcuta. Universidad Francisco de Paula Santander. Facultad de formación tecnológica. Tecnología química.1992.

6. Instituto Colombiano de Geología y Minería. INGEOMINAS. Recursos Minerales de Colombia. Publicaciones especiales del INGEOMINAS. $2^{\text {a }}$ ed. Tomo II. Bogotá: INGEOMINAS, 1998, $350 \mathrm{p}$.

7. Shaw K. Refractories and their Uses. London: Applied Science Publishers LTD., 6 p. 1972.

8. Stephen, C. Carniglia And Gordon L. Barna. Handbook of Industrial Refractories Technology. Principles, Types, Properties and Applications. Published by noyes Publications, 1992.

9. Semler C. Material classification of refractories. Handout of ceramic engineering 633. Columbus: The Ohio State University, 1985.

10. Norton F. H., Refractarios, 4a ed. Editorial Blume. Madrid., 1971. 467p

11. Álvarez-Estrada D; De Aza Pendas S. Ladrillos refractarios básicos aglomerados químicamente en frío. Boletín de la sociedad española de cerámica y vidrio VOL. 5 - N. ${ }^{\circ}$ 5. 1966.

12. Audin C. Resistencia de los refractarios al choque térmico. I: Aproximación termoelástica y criterio de balance energético. Instituto de Cerámica y Vidrio (CSIC). 28500 Madrid (España). 1993.

13. Rendtorff N; Garrido L; Aglietti E. Choque Térmico de Materiales Refractarios: Comportamiento, Evaluación y Ensayos. CERAMICA Y CRISTAL 139 - MARZO 2007 - ISSN 0325 0229 www.ceramicaycristal.com.ar

14. H de Aza, A. Corrosión de materiales refractarios por escorias y vidrios fundidos. Boletín de la sociedad española de cerámica y vidrio. 35 [2] 87-101 (1996)

15. Castillo S, Rojo G. Evolución de los materiales refractarios en cuchara. Refractarios de alta alúmina. Boletín de la sociedad española de cerámica y vidrio Vol. 28 - Núm. 5. 1989

16. NTC 674. Método de ensayo para determinar porosidad aparente, absorción de agua, grave- 
dad específica aparente y densidad aparente por agua en ebullición de ladrillos refractarios y piezas refractarias quemadas.

17. NTC 4321-4. Ingeniería civil y arquitectura. Baldosas cerámicas. Parte 4. Método de ensayo para determinar el módulo de rotura y la resistencia a la flexión.

18. NTC 706. Método de ensayo para determinar el cono pirométrico equivalente (CPE) de materiales refractarios silicoaluminosos y de alta alúmina. 